\title{
Friction Effect Analysis of a DC Motor
}

\author{
Ivan Virgala*, Peter Frankovský, Mária Kenderová \\ Department of applied mechanics and mechatronics, Technical university of Košice / Faculty of mechanical engineering, Košice, \\ Slovakia \\ *Corresponding author: ivan.virgala@tuke.sk
}

\author{
Received December 21, 2012; Revised January 13, 2013; Accepted February 26, 2013
}

\begin{abstract}
Precision positioning systems are fundamental components in industrial machines such as machine tools, measuring machines and semiconductor manufacturing systems. The performance of the machines depends on the positioning systems. For precise positioning are often used DC motors. The paper deals with experimental method of DC motor friction identification. The aim of the paper is determining of suitable way of DC motor friction modeling. At first the basic static friction models are introduced and linear mathematical model of DC motor is established. DC motor friction identification is by means of experimental method done using Newton's mechanics. For experiment PITTMAN 9413 motor with encoder is used. For measuring of friction torque the measuring I/O card is used which cooperates with Matlab / Simulink by Real Time Toolbox. Then the mathematical model is simulated and compared with real model of DC motor. In the conclusion the effects of particular friction models affecting DC motor are described and the best way of friction modeling for DC motor is determined.
\end{abstract}

Keywords: DC motor, friction, identification, Simulink, Stribeck

\section{Introduction}

Nowadays there are a lot of tasks in mechatronics which concern precise positioning in mechanisms. Mostly a friction phenomenon plays large problem in these tasks and many times it has to be compensated. The friction can be compensated be various ways, for example by design solution, by lubricant or by suitable friction compensation method. The friction is a phenomenon which is not easily to describe exactly. For these purposes there were a lot of friction models and compensating schemes described.

Modeling and identification of mechanical systems constitute an essential stage in practical control design and applications. Controllers commanding systems that operate at varying conditions or require high precision operation raise the need for a non-linear approach in modeling and identification. Most mechanical systems used in industry are composed of masses moving under the action of position and velocity dependent forces. These forces exhibit nonlinear behavior in certain regions of operation [1].

DC motors, as components of electromechanical systems, are widely used as actuating elements in industrial applications for their advantages of easy speed and position control and wide adjustability range. Consequently, examination of DC motor behavior constitutes a useful effort for analysis and control of many practical applications. In modeling a DC motor connected to a load via a shaft, the general approach is to neglect the nonlinear effects and build a linear transfer function representation for the input-output relationship of the DC motor and the load it drives $[2,3,11]$.

In this study a friction of DC motor is investigated. A mathematical model of a DC motor is well know and if we know to describe a friction in motor we can easily control angular velocity or angular position.

\section{Static Friction Models}

Friction is the tangential reaction force between two surfaces which are in the contact. In dry sliding contacts between flat surfaces friction can be modeled as elastic and plastic deformation forces of microscopical asperities in contacts. In dry rolling contact, friction is the result of a non-symmetric pressure distribution in the contact. Other physical mechanisms appear when lubricant is added to the contact. For low velocities, the lubricant acts as a surface film, where the shear strength determines the friction. At higher velocities at low pressures a fluid layer of lubricant is built up in the surface due to hydrodynamic effects $[13,14]$.

The behavior of friction has been extensively examined during the $20^{\text {th }}$ century. The experiments have been performed under idealized conditions with clean surfaces and for stationary conditions, e.g. constant velocity.

\subsection{Static Friction}

Static friction is the friction when the two surfaces are sticking. The force required to overcome the static friction and initiate motion is called the breakaway force. The static friction is usually larger than Coulomb friction force.

\subsection{Coulomb Friction}

Coulomb friction is independent of velocity and is always present. This friction component is only dependent on the direction of motion, in such way that it is in the direction opposite to the velocity. The magnitude of 
Coulomb friction depends on the properties of the surfaces in contact and the normal force. Coulomb friction is also known as kinetic friction [12].

\subsection{Viscous Friction}

Viscous friction is dependent of the velocity. At zero velocity the viscous friction is zero and the viscous component increases with the increase of velocity.

\subsection{Stribeck Friction}

Stribeck friction arises from the usage of fluid lubrication. At low velocity the friction will decrease with the increase velocity. Stribeck friction is usually expressed by following equation [15]:

$$
F_{f}(v)=F_{C}+\left(F_{S}-F_{C}\right) e^{-\left|\frac{v}{v_{S}}\right|^{\delta}}+F_{V} v
$$

where $F_{C}, F_{S}, v_{S}, F_{V} v$ are Coulomb friction, static friction, stribeck velocity and viscous friction, respectively. The Stribeck friction is in the Figure 1 shown.

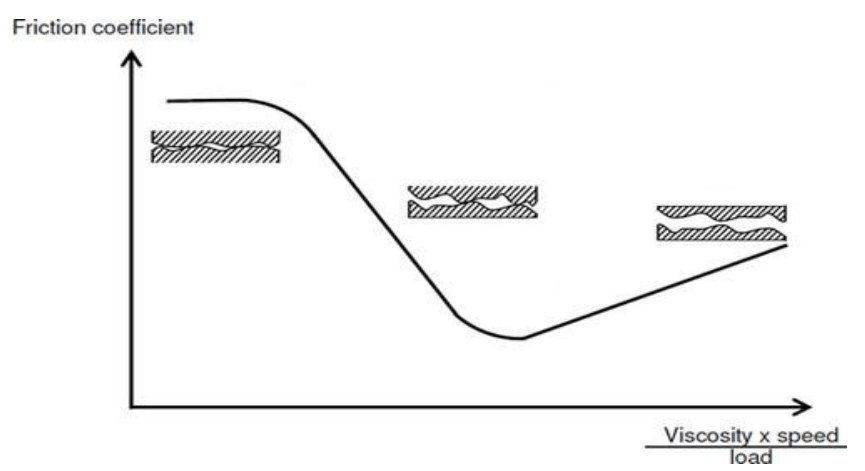

Figure 1. Stribeck friction

\section{DC Motor Mathematical Model}

A DC motor consists of two sub-processes: electrical and mechanical. The electrical sub-process consists of armature inductance, armature resistance and the magnetic flux of the stator. A second sub-process in the motor is a mechanical one. It consists of the inertia of the motor and a load. The difference in motor speed is caused by the electromagnetic moment generated by the amplifier current, load and friction of the motor [4].

The main advantages of the DC motors are easy speed or position control and wide adjustable range to follow a predetermined speed or position trajectory under load. These have been extensively used in several industrial applications $[5,6,7,8,9,10]$.

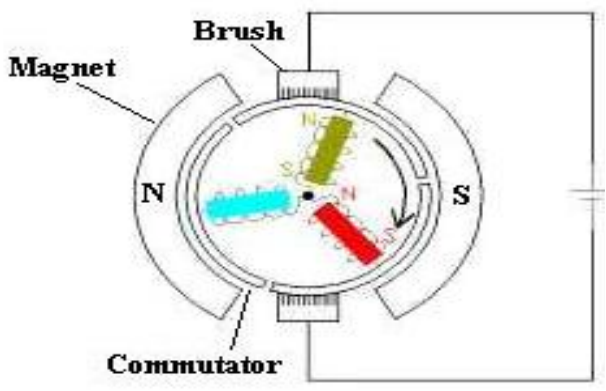

Figure 2. Permanent magnet DC motor
One of the common used actuator in control systems is a DC motor. The Figure 3 shows simplified electrical circuit of DC motor and mechanical model of a rotor.
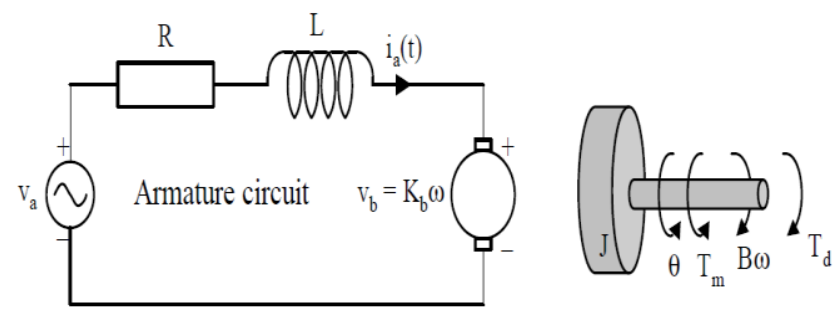

Figure 3. DC motor

For the armature of DC motor we will use (PITTMAN 9413) the following parameters values are:

$\mathrm{R}=$ armature resistance $=5.35 \Omega$

$\mathrm{L}=$ armature inductance $=3.93 \mathrm{mH}$

$\mathrm{J}=$ moment of inertia $=2.75 \mathrm{e}^{-6} \mathrm{kgm}^{2}$

$\mathrm{K}_{\mathrm{t}}=$ motor torque constant $=0.0316 \mathrm{Nm} / \mathrm{A}$

$\mathrm{K}_{\mathrm{e}}=$ back emf constant $=0.0316 \mathrm{Vrad} / \mathrm{s}$

$\mathrm{B}=$ viscous friction (will be identified by experiment)

$\mathrm{T}_{\mathrm{m}}=$ motor torque

The mathematical model of DC motor is expressed by following equations:

$$
\begin{gathered}
J \frac{d \omega(t)}{d t}+B \omega(t)=T_{m}(t) \\
L \frac{d i_{a}(t)}{d t}+R i_{a}(t)=v_{a}(t)-v_{b}(t) \\
v_{b}=K_{e} \omega(t) \\
T_{m}(t)=K_{t} i_{a}(t)
\end{gathered}
$$

By means of Laplace transformation we can obtain transfer function of DC motor.

$$
\frac{\Omega(s)}{V_{a}(s)}=\frac{K_{t} K_{e}}{(J s+B)(L s+R)+K_{t} K_{e}}
$$

The mathematical model can be expressed by block diagram according the Figure 4.

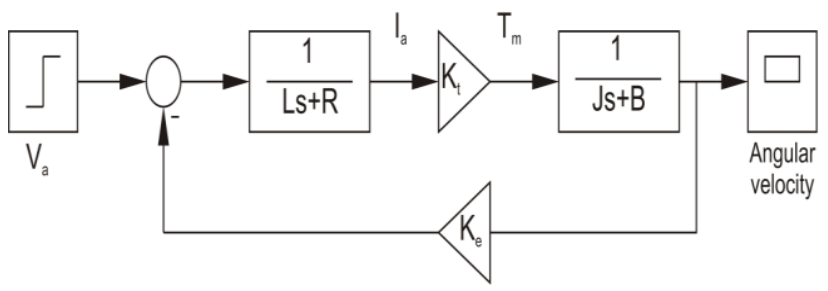

Figure 4. Block diagram of DC motor

Accurate model building is a crucial stage in practical control problems. An adequately developed system model is essential for reliability of the designed control. When the plant has uncertainties or time dependencies, or cannot be parameterized, a model for the system may be hard to obtain. For such systems, the system parameters should be determined using system identification techniques [3].

If all the variables of motor are know the experiment for friction identification can be done. 


\section{Experiments and Simulations}

Consider the DC motor motion equation in general.

$$
J \frac{d \omega(t)}{d t}=T_{m}(t)-T_{f}
$$

where $T_{f}$ is motor friction in general. When angular velocity has constant value, the left side of equation equals zero and motor torque equals to friction torque.

$$
T_{m}(t)=T_{f}
$$

Now the motor friction measuring task changes to the motor torque measuring, of course for constant angular velocity. Using DC motor without any load with voltage supply we reach angular velocity, what is by motor encoder measured.

\subsection{Experiment}

The experiment is by means of input-output measuring card MF624 done. MF624 has a several digital inputs/outputs, analog inputs/outputs and encoders. Limitation of inputs and outputs signals is $+-10 \mathrm{~V}$. The measuring card cooperate with software Matlab / Simulink through Real Time Toolbox.

The wiring scheme is in the Figure 5 shown.

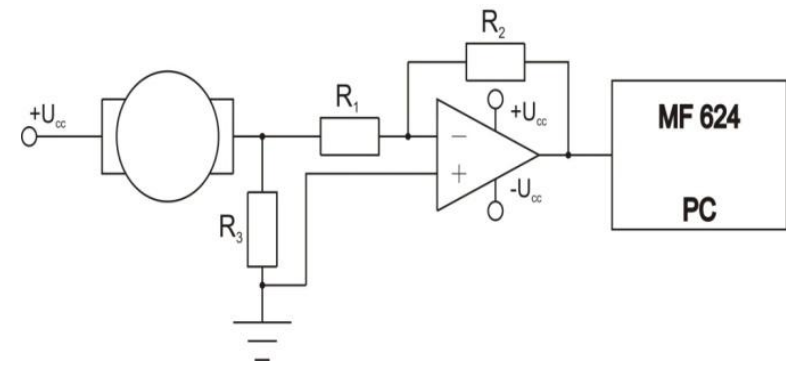

Figure 5. Wiring scheme

The measuring card can work only with voltage signal therefore we have to measure voltage change on the resistor $R_{3}$. According to Ohm's law can be actual current expressed and by measuring of actual current we can express actual motor torque which equals to the friction torque. Photo from experiment in the Figure 6 is shown.

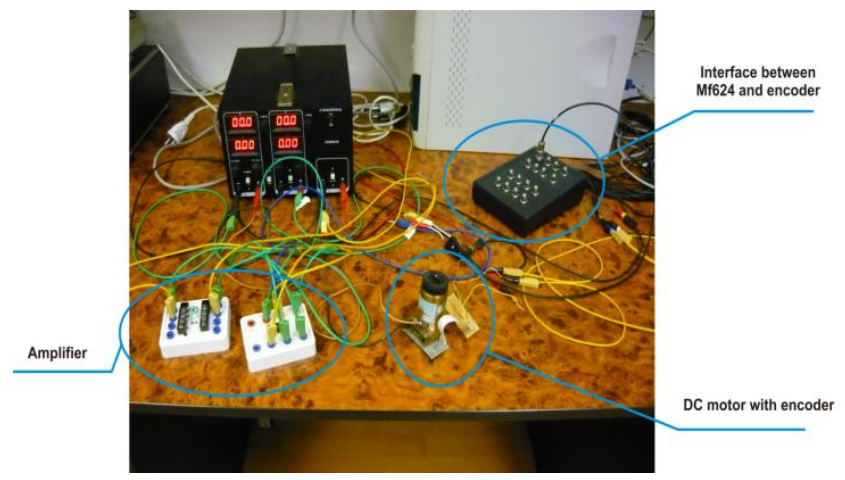

Figure 6. Experiment

At first the static friction of dc motor is determined. The motor voltage supply increases to the moment when the motor shaft starts rotate. The maximum value of motor torque for stationary motor shaft presents static friction torque. For static friction of motor were done ten measurements. The example of measurement in the Figure 7 is shown.
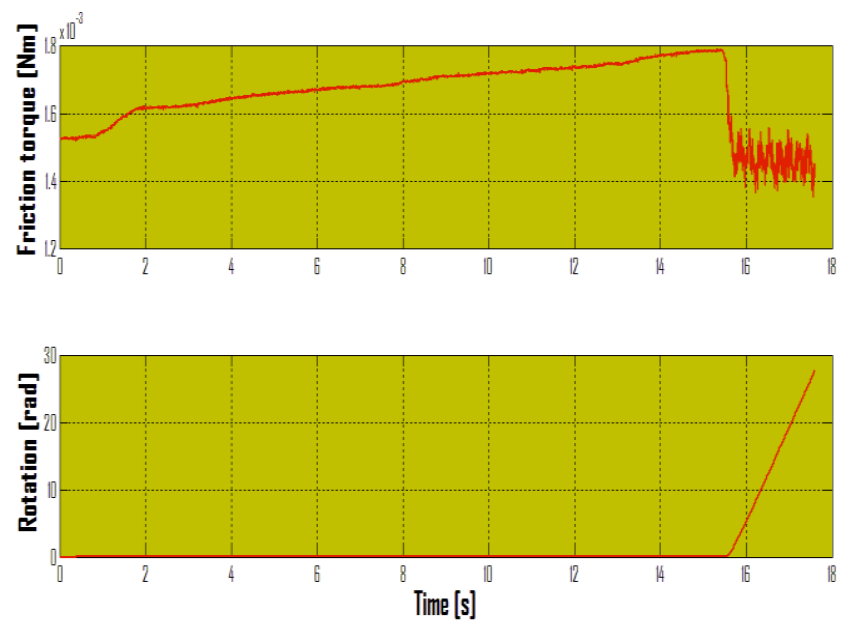

Figure 7. Measurement of the DC motor static friction

As can be in the Figure 7 seen, the motor friction decreases when the shaft starts rotate what indicates Stribeck effect.

On the next figure is shown measured DC motor torque for constant angular velocity.
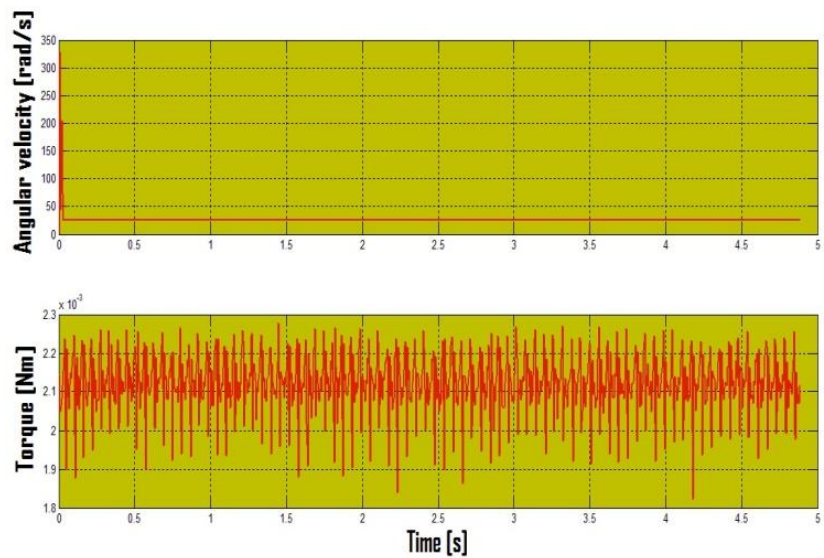

Figure 8. DC motor torque for constant angular velocity

For constant velocity is determined DC motor torque by computation of torque average value.

Next measurement is for motor in motion. Motor supply voltage gradually increases with step $0.3 \mathrm{~V}$ up to the value $19 \mathrm{~V}$. The results of measurements in the figure 9 are shown.

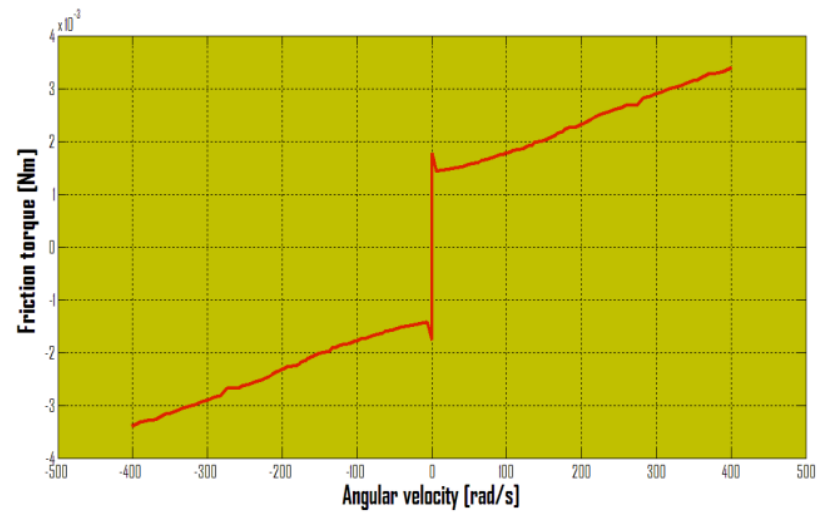

Figure 9. Friction characteristics of DC motor 
In the Figure 9 can be seen Stribeck friction. From the static friction the friction decreases up to Stribeck velocity and then mainly the viscous friction occurs.

Now the mathematical model can be verified with measured values.

\subsection{Simulation and Comparison with Experiment}

This section discusses with ways of friction modeling. At first sight the friction characteristics appear like linear increasing with significantly slope. So, at first we can model friction like viscous friction. Neglecting the static friction we get linear equation:

$$
y(x)=0.0000049804 x
$$

Assuming that DC motor stall torque is $0.1102 \mathrm{Nm}$ (from datasheet) the slope of viscous part of friction is very small. The curve with very small slope should be replaced by straight line which presents Coulomb friction model with value $0.0025 \mathrm{Nm}$, see Figure 10 .

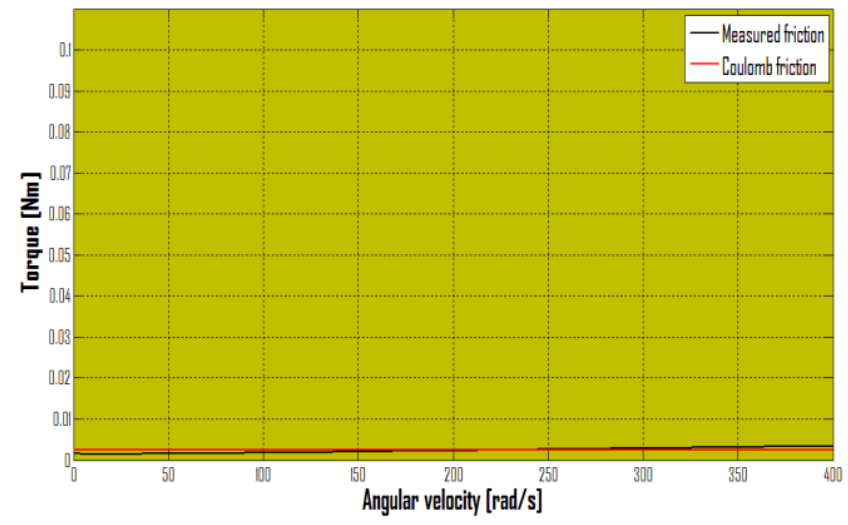

Figure 10. Coulomb friction in comparison with measured friction

In the Figure 11 the all three frictions are shown.

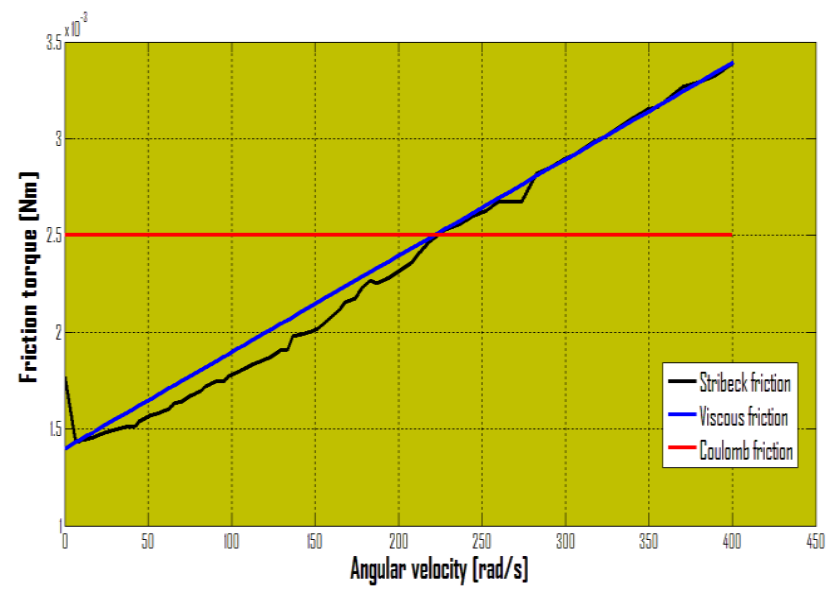

Figure 11. Stribeck, viscous and Coulomb friction

After DC motor friction identification can be verification done in software Matlab / Simulink. Verification is based on mathematical model with assuming three different friction courses. The first is model with Stribeck friction, second is model with viscous friction and third is model with Coulomb friction. The models are compared with experiment where as input variable is DC motor voltage supply (in the simulation represented by step block) and as output variable is angular velocity. The results in the figures 12-14 are shown.

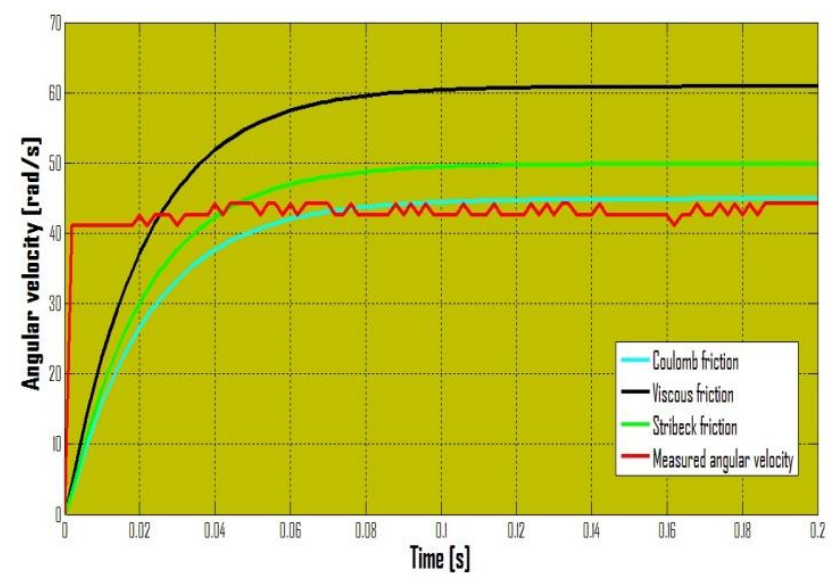

Figure 12. DC motor angular velocity with different friction models voltage supply $-2 \mathrm{~V}$

Closest to the measured angular velocity is model with Coulomb friction. The next is Stribeck friction. Interestingly, that viscous friction which is very similar to Stribeck friction, see Figure 11, gives less precise result in comparison with Coulomb friction.

On the next figure the same measurement is done with the voltage supply $4 \mathrm{~V}$.

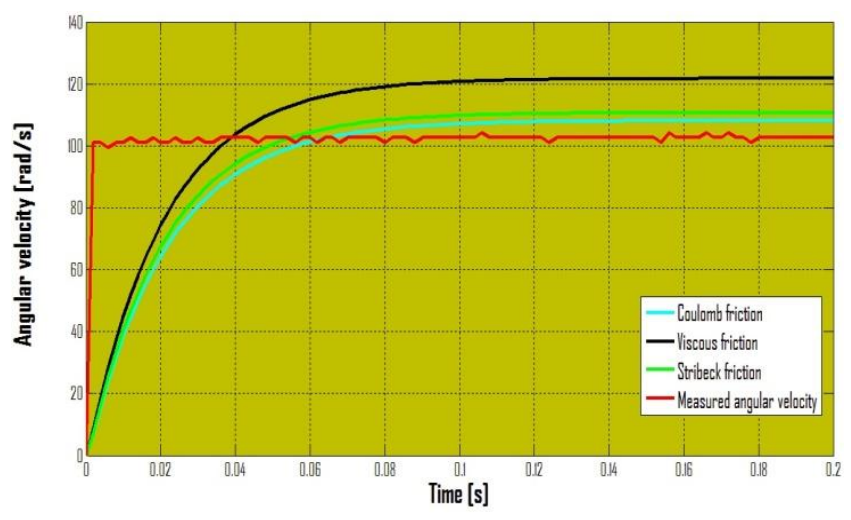

Figure 13. DC motor angular velocity with different friction models voltage supply $-4 \mathrm{~V}$

Now for voltage supply $-4 \mathrm{~V}$, the model with Coulomb friction is not so close to the measured angular velocity as in the case with $2 \mathrm{~V}$.

In the next figure the voltage supply is $6 \mathrm{~V}$.

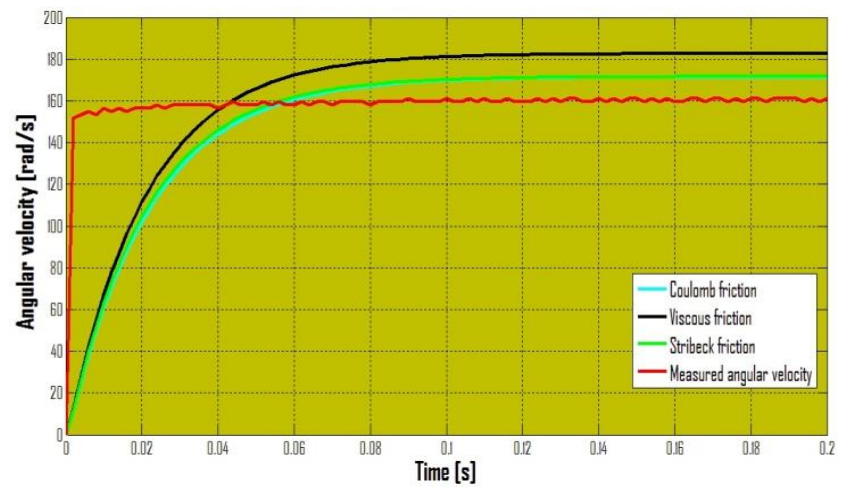

Figure 14. DC motor angular velocity with different friction models voltage supply - $6 \mathrm{~V}$ 
From the Figure 14 is obvious that Coulomb friction is almost same as Stribeck friction. From the last three simulations it can be seen that the higher voltage supply is the bigger distance appears between measured angular velocity and angular velocity of model with assuming Coulomb friction. From this reason it is not suitable solution to use Coulomb friction model to modeling DC motor mathematical model.

Nevertheless, that viscous friction model gives better mathematical model because of its linearity, for more precisely results it should be used Stribeck friction model, which is very close to measured angular velocity.

\section{Conclusion}

The problem of accurate control of electromechanical systems is very important in many industrial applications such devices, e.g. motors or essential parts of positioning / tracking systems. A friction, occurring in moving mechanisms, causes failures especially during precise position regulation. Friction was described by several friction models in the past. In the paper the DC motor (PITTMAN 9413) friction is identified by experimental method using measuring card MF624 which cooperate with Matlab / Simulink. The result shows that Coulomb friction model gives better result in comparison with viscous friction model but the best result gives mathematical model of DC motor with considering Stribeck friction. Coulomb and Stribeck friction model causes non-linearities in model in comparison with viscous friction model but gives more precisely result. For tasks, where DC motor is used for precise positioning should be used more complicated model of friction like Stribeck friction.

\section{Acknowledgement}

The authors would like to thank to Slovak Grant Agency - project VEGA 1/1205/12 "Numerical modeling of mechatronic systems". This contribution is also result of the project APVV-0091-11 "Using of methods of experimental and numerical modeling for increasing of competitiveness and innovation of mechanical and mechatronical systems." This contribution is also the result of the grant project of Tatra Banka - "LocoSnake robotic mechanisms motion with steering software support".

\section{References}

[1] T. Kara, I. Eker, "Nonlinear modeling and identification of a DC motor for bidirectional operation with real time experiments", Energy and Management, Elsevier, 2003.

[2] J. Horng, "Neural adaptive tracking control of a DC motor", Informat Sci, 1999.

[3] T. Söderström, T. Stoica, "System identification". Cambridge, UK Prentice Hall, 1989.

[4] T. Tjahjowidodo, F. Al-Bender, H. Van Brussel, "Friction identification and compensation in a DC motor", Mechanical Engineering Department, Heverlee, Belgium.

[5] S. E. Lyshevski, Nonlinear control of mechatronic systems with permanent-magnet DC motors. Mechatronics, Vol. 9, pp 539-552, 1999.

[6] J. O. Jang and G. J. Jeon, A parallel neuro-controller for DC motors containing nonlinear friction, Neurocomputing, Vol. 30, pp 233-248, 2000.

[7] J. H. Horng, Neural adaptive tracking control of a DC motor, Information Sciences, Vol.118, pp 1-13, 1999.

[8] M. J. Chung and D. G. Gweon, Modelling of the armature slotting effect in the magnetic field distribution of a linear permanent magnet motors, Electrical Eng, Vol. 84, pp 101-108, 2002.

[9] K. Ziaei and N. Sepehri, Modelling and identification of electrohydraulic servos. Mechatronics, Vol. 10, pp 761-772, 2000.

[10] Y. Yavin and P. D. Kemp, Modelling and control of the motion of a rolling disk: effect of the motor dynamics on the dynamical model, Comput. Methods in Appl Mec and Eng, Vol.188, pp 613624, 2000.

[11] M. Štamborská, "Determination of stress fields from measured relative deformation fileds using digital image correlation", I. Workshop of young researchers, VŠB - TUO, ISBN 978-80-2482885-5, 2012

[12] H. Olsson, K. J. Åström, C. Canudas de Wit, M. Gäfvert, and P. Lischinsky, "Friction models and friction compensation", European journal of control, No. 4, pp. 176-195, December 1998.

[13] C. Iurian, F. Ikhouane, J. Rodellar, R. Grinó, "Identification of a system with dry friction", University of Barcelona, September 2005.

[14] B. Amstrong, "Stick slip and control in low-speed motion", IEEE transaction on automatic control, vol. 38, No. 10, 1993.

[15] R. Hensen, "Controlled mechanical systems with friction", Technische Universiteit Eindhoven, ISBN 90-386-2693-2, 2002. 